\title{
Fragmentation function measurements
}

\author{
Ralf Seidl* \\ RIKEN, Japan \\ E-mail: rseidleriken.jp
}

\begin{abstract}
Fragmentation functions describe how quasi-free, high energetic partons fragment into a number of confined final state hadrons. Traditionally, only inclusive hadron production in $e^{+} e^{-}$annihilation was studied as a function of the hadrons scaled momentum or energy and mostly at collision energies around the $\mathrm{Z}$ mass. Recently, both $\mathrm{B}$ factories published identified hadron data near the $\Upsilon(4 S)$ resonance which allow to extract the gluon fragmentation from the scaling violation between these energies. Also new precision data from semi-inclusive deep inelastic scattering and proton-proton collisions is being used in global extractions on fragmentation functions. Furthermore spin dependent fragmentation functions were extracted in recent years and are crucial in the understanding of various previously puzzling transverse spin phenomena.
\end{abstract}

XV International Conference on Hadron Spectroscopy-Hadron 2013

4-8 November 2013

Nara, Japan

\footnotetext{
${ }^{*}$ Speaker.
} 


\section{Introduction}

The transition of quasi-free, high energetic partons into confined hadrons cannot be described from first principle QCD as the related parton and hadron masses are typically too low to apply perturbative QCD. These fragmentation functions (FF) therefore need to be measured experimentally similarily to the parton distribution functions (PDFs) in the nucleon. Their definition is also rather similar as for example the fragmentation function $D_{1, q}^{h}(z, Q)$ describes the number density of producing a hadron $\mathrm{h}$ from a parton $\mathrm{q}$ with fractional energy $z=E_{h} / E_{q}$ and at an energy scale $\mathrm{Q}$. Fragmentation functions are defined in the quark-parton model as bi-local operators and in principle various individual fragmentation functions can be projected out given the corresponding Dirac matrix combinations, but in practice only vector (momentum fraction), pseudo-vector (longitudinally polarized) and tensor (transversely polarized fragmentation) components are relevant to date. As the calculation of fragmentation functions involves at least one final state hadron they are not directly calculable on the Lattice in contrast to parton distribution functions.

\section{Unpolarized fragmentation}

Traditionally fragmentation functions were extracted only in $e^{+} e^{-}$annihilation as here the cleanest initial state is available without any hadrons and predominantly only a back-to-back quark-antiquark pair as outgoing partons. The fragmentation into final state hadrons can therefore be cleanly studied by measuring the inclusive yield of single hadrons: $e^{+} e^{-} \rightarrow h X$ where $\mathrm{X}$ stands for the undetected final state. This has been performed at various $e^{+} e^{-}$colliders and the majority of the existing fragmentation data comes from SLD and LEP experiments at or around the $Z^{0}$ resonance, see [1] for an overview of the $e^{+} e^{-}$fragmentation world data. However, as the gluon fragmentation only enters in $e^{+} e^{-}$annihilation at the nextto-leading order level in the strong coupling, it is only accessible via the scaling violation at different energy scales. Until recently, very little fragmentation data was available at relatively low scales compared to the $Z^{0}$

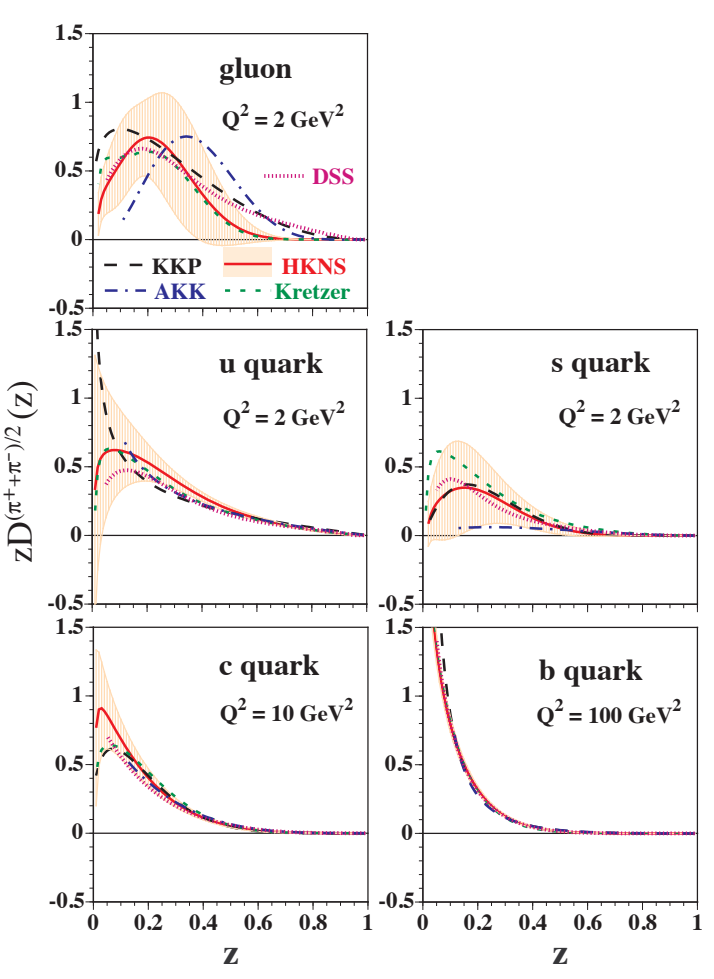

Figure 1: Current uncertainties of various global QCD fits for pion fragmentation as a function of $z$. resonance and therefore the gluon fragmentation function from $e^{+} e^{-}$annihilation data alone was only poorly constrained. Also very little information at high fractional energies $z$ existed in general and therefore also those high- $z$ regions were not very well constrained. Various theory groups perform global types of analysis on the ex- 
isting world data in order to extract fragmentation functions [2, 3, 4], but just using the previously available data not all fragmentation functions are well constrained, yet, see for example Fig. 1 The two high-statistics B factory experiments BaBar and Belle can provide precision data at comparatively low scales close to the Upsilon(4S) resonance and center-of-mass energies of $\sqrt{s}=10.52$ $\mathrm{GeV}$ (10.54 GeV in BaBar) and out to very high fractional energies. Both experiments have just published either identified charged pion, kaon and proton multiplicites [5] or differential cross sections for identifies charged pions and kaons [6]. The Belle results are for example shown in Fig. 2 where one can see, that the statistical and systematic uncertainties remain very small to the highest fractional energies and are generally on the \% level. The addition of these two measurements will allow significant improvements of in particular the gluon fragemantation and also the kaon fragmentation functions in an updated global QCD analysis. Several prospects of the improvements have been shown by the involved theory groups at a recent fragmentation function workshop [7]. However, as the treatment of weak decays and in particular initial state photon radiation was different between the two B-factory measurements they are not as easily comparable in the global fits and some quantitative understanding of the different treatments needs to extracted.

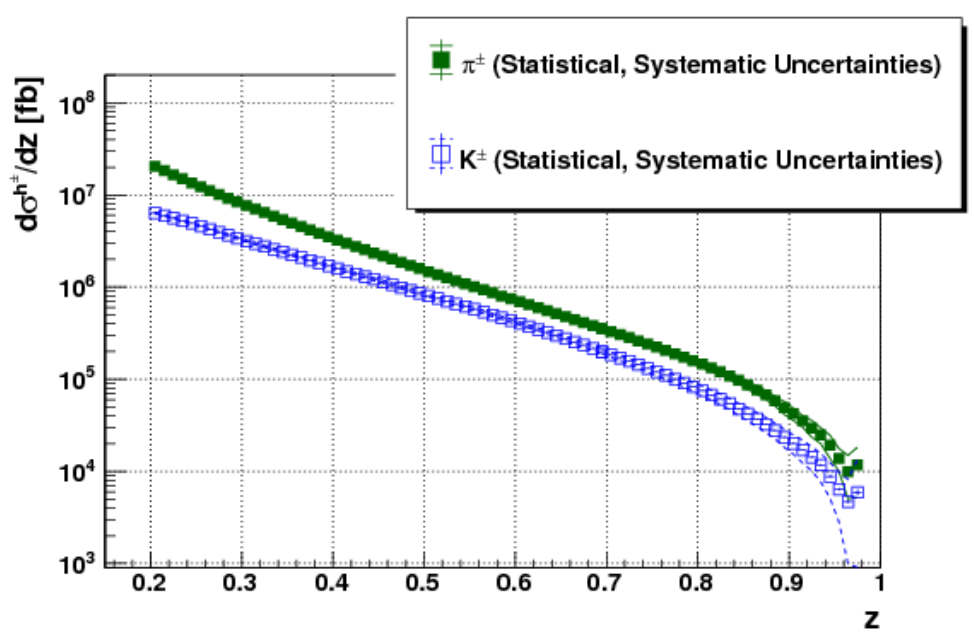

Figure 2: Pion and kaon differential cross sections as a function of $z$ from the Belle experiment.

One shortcoming of only using $e^{+} e^{-}$annihilation data is, that apart from the access to the gluon fragmentation only via the evolution, also quark and anti-quark fragmentation cannot be distinguished in inclusive single hadron measurements and only the sum of favored and disfavored fragmentation is accessible. Favored fragmentation describes the fragmentation of a quark into a hadron with the same flavor as valence quark and disfavored that of a different flavors, for example $u \rightarrow \pi^{+}$vs $u \rightarrow \pi^{-}$. As unpolarized parton-distribution functions at intermediate parton momentum fractions $x$ are reasonably well known, other processes such as semi-inclusive deep inelastics lepton-nucleon scattering (SIDIS) and hadron production in nucleon-nucleon collisions can be used as well to obtain information on fragmentation functions. The HERMES experiment has published precise hadron multiplicities for pions and kaons from both a proton and deuteron target [8] and recently also preliminary pion and kaon multiplicities from the COMPASS experiment 
on a LiD target became available [9]. These measurements will provide important information in disentangeling favored and disfavored fragmentation functions into pions and kaons. In addition also recent measurments of hadron cross sections at RHIC [10, 11] and LHC [12] provide useful information as in hadron-hadron collisions the hard processes are dominated by gluon interactions and thus gluon fragmentation is already accessible at leading order in the strong coupling. Together with this new information one can expect substantially improved global fits of the fragmentatation functions in the near future.

Another aspect of unpolarized fagmentation functions which has gotten quite some interest recently is the transverse momentum generated in fragmentation. It is important in the relation to various transverse spin dependent quantities and the potential saturation of the gluon PDF at very low x. While some simple Gaussian dependence is even included in various models including JETSET [13] little detailed data has been available since recently. So far both HERMES [8] and COMPASS [14] have provided transverse momentum dependent results, see Fig. 3, but this transverse momentum is convoluted with transverse momentum the partons already carry in the nucleon. Nevertheless a first attempt at extracting the $\mathrm{x}$ and $\mathrm{z}$ dependence of intrinsic and fragmentation transverse momenta have been performed [15]. Results from the B factories on explicit fragmentation transverse momenta are expected soon as well.

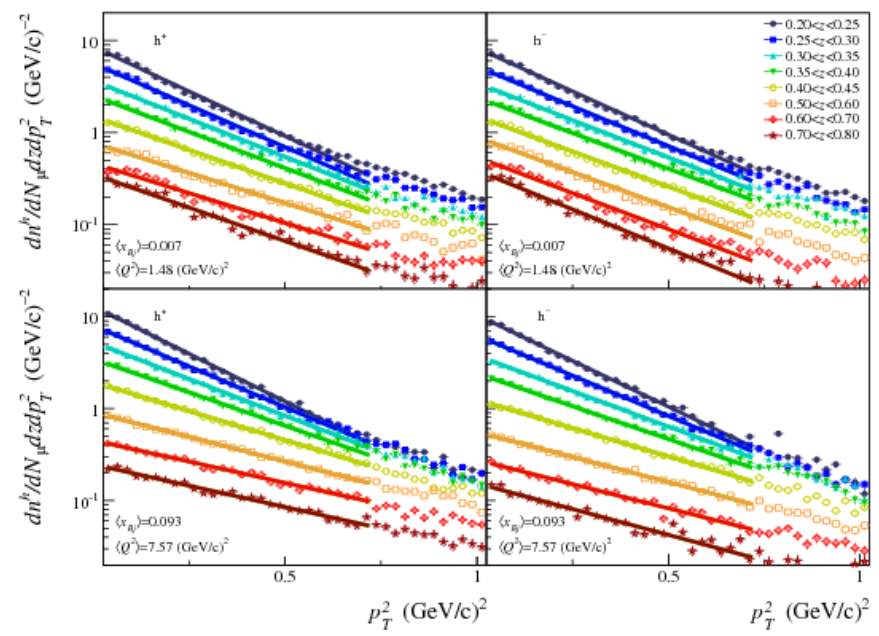

Figure 3: Hadron multiplicities in bins of $x$ and $z$ as a function of the square of the transverse momentum for positive (left) and negative (right) charges. A Gaussing fit to the lower transverse momenta is also displayed.

\section{Polarized fragmentation functions}

Spin dependent fragmentation functions were first introduced in order to explain the unexpectedly large hadron single spin asymmetries in transversely polarized proton-proton collisions [16]. Together with a nonzero quark transversity distribution, the so-called Collins effect would exhibit a left-right asymmetry of the hadron yield around the direction of a polarized quark and with respect to the quark spin. This effect has first been directly found in the HERMES experiment [17] and later been confirmed by COMPASS[18]. In $e^{+} e^{-}$annihilation only the spin-dependent fragmentation 
function without the quark transversity distribution can be observed and asymmetries of pion pairs related to the Collins fragmentation function were extracted by the Belle experiment [19]. Here one accesses a product of two Collins fragmentation functions normalized by their unpolarized counterparts. It was found, that the Collins fragmentation function is large and rises as a function of $z$. These results have recently been confirmed by BaBar [20] who also include the transverse momentum dependence of the Collins function as shown in Fig. 4. Generally the transvese momentum dependence at low scales such as measured in the SIDIS experiments is expected to have Gaussian widths significantly smaller than the BaBar results, where no clear peak can be identified. However, this could be an aspect of the transverse momentum dependent QCD evolution with significantly broader widths at scales as high as in the B factories.

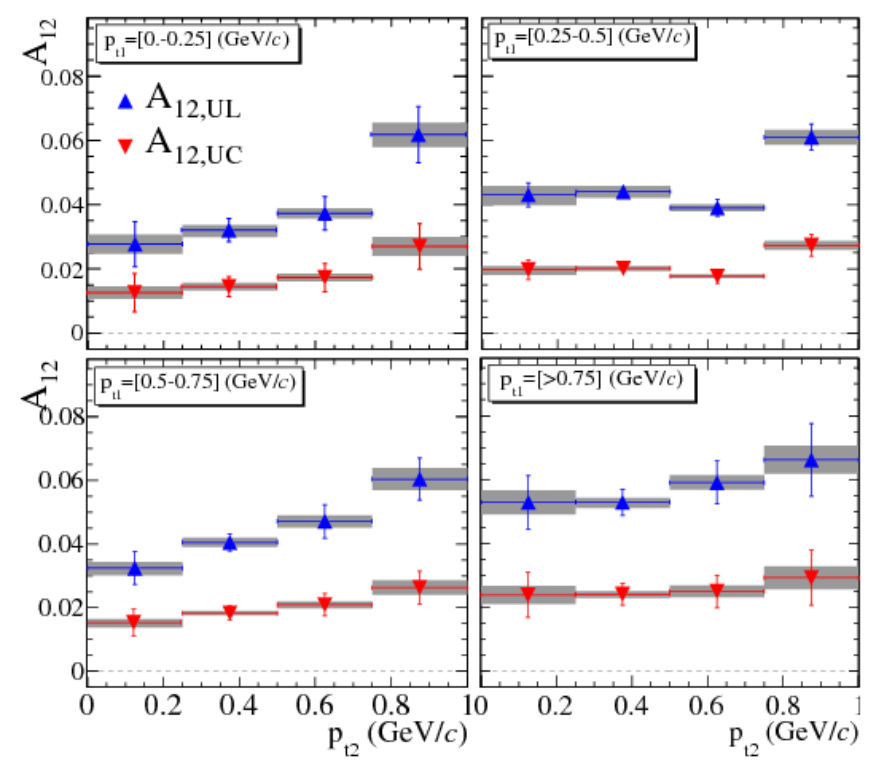

Figure 4: $\cos \left(\phi_{1}+\phi_{2}\right)$ moments related to the product of two Collins fragmentation fucutions as a function of the transverse momentum of one hadron in bins of the other hadron's transvers momentum.

Using the Collins related measurements in $e^{+} e^{-}$annihilation and transversely polarized SIDIS it was for the first time possible to extract the quark transversity distribution [21]. The extracted transversity distribution is shown in Fig. 5 where one sees sizeable up and down quark transversity distributions with the down quark approaching the Soffer bound [22] at high (but mostly not experimentally covered) $x$.

However, as still not too much information about the explicit transverse momentum dependence of polarized and unpolarized fragmeantion functions is known, this transversity extraction relies on various assumptions. Furthermore also the scale dependence of the Collins function is not yet known and was assumed to be as that of unpolarized fragmentation. A second, independent access to transverseity exists which allows to test, whether these assumptions are valid. The so-called interference fragmentation function [23] does not depend on the transverse momentum and therefore its scale dependence is known. However also here some assumptions were necessary in order to extract transversity from the HERMES [24], COMPASS [25] and Belle[26] data. Surprisingly 

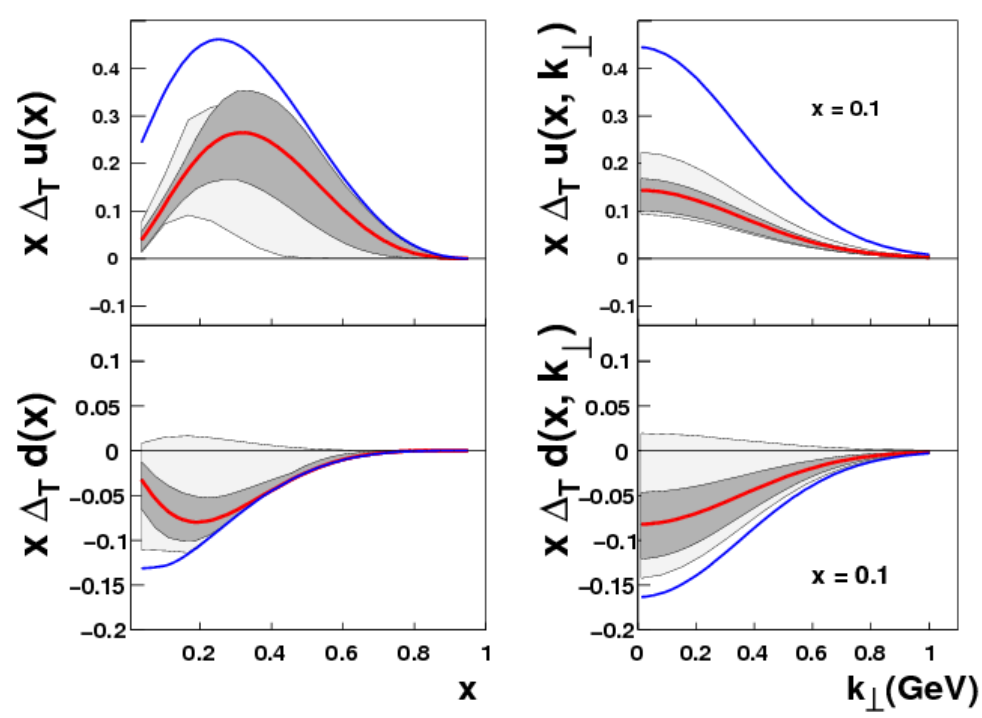

Figure 5: Quark transversity distributions (red line) for $u$ quarks (top) and $d$ quarks as a function of the parton momentum fraction $x$ (left) and the intrinsic transverse momentum $k_{T}$ (right). The light and dark grey bands show previously obtained and recent uncertainties respectively and the blue curve displays the Soffer bound.

this extraction [27] coincides very well with the Collins based extraction. Further measurements related to Collins and interference fragmentation functions are ongoing in the B factories, SIDIS and pp experiments which will allow a better determination of the transversity distribution and a more detailed flavor decomposition. It is expected that an eventual electron-ion collider will allow to not only extract the flavor decomposition of transversity but also the corresponding tensor charges which require integration over $x$.

\section{References}

[1] J. Beringer et al. [Particle Data Group Collaboration], Phys. Rev. D 86, 010001 (2012).

[2] M. Hirai, S. Kumano, T. -H. Nagai and K. Sudoh, Phys. Rev. D 75, 094009 (2007)

[3] D. de Florian, R. Sassot and M. Stratmann, Phys. Rev. D 75, 114010 (2007). M. Epele, R. Llubaroff, R. Sassot and M. Stratmann, Phys. Rev. D 86, 074028 (2012).

[4] S. Albino, B. A. Kniehl and G. Kramer, Nucl. Phys. B 803, 42 (2008).

[5] J. P. Lees et al. [BaBar Collaboration], Phys. Rev. D 88, 032011 (2013).

[6] M. Leitgab et al. [Belle Collaboration], Phys. Rev. Lett. 111, 062002 (2013).

[7] Indiana-Illinois Workshop on Fragmentation Functions, http://www.indiana.edu/ ffwrkshp/index.html 
[8] A. Airapetian et al. [HERMES Collaboration], Phys. Rev. D 87, 074029 (2013).

[9] N. Makke, arXiv:1307.3407.

[10] G. Agakishiev et al. [STAR Collaboration], Phys. Rev. Lett. 108, 072302 (2012).

[11] A. Adare et al. [PHENIX Collaboration], Phys. Rev. D 76, 051106 (2007).

[12] B. B. Abelev et al. [ALICE Collaboration], Eur. Phys. J. C 73, 2662 (2013).

[13] T. Sjostrand, S. Mrenna and P. Z. Skands, JHEP 0605, 026 (2006).

[14] C. Adolph et al. [COMPASS Collaboration], Eur. Phys. J. C 73, 2531 (2013).

[15] A. Signori, A. Bacchetta, M. Radici and G. Schnell, JHEP 1311, 194 (2013).

[16] J. C. Collins, Nucl. Phys. B396 (1993) 161.

[17] A. Airapetian et al. [HERMES Collaboration], Phys. Rev. Lett. 94, 012002 (2005).

[18] V. Y. Alexakhin et al. [COMPASS Collaboration], Phys. Rev. Lett. 94, 202002 (2005). C. Adolph et al. [COMPASS Collaboration], Phys. Lett. B 717, 376 (2012).

[19] K. Abe et al. [Belle Collaboration], Phys. Rev. Lett. 96, 232002 (2006). R. Seidl et al. [Belle Collaboration], Phys. Rev. D 78, 032011 (2008) [Erratum-ibid. D 86, 039905 (2012)].

[20] J. P. Lees et al. [BaBar Collaboration], arXiv:1309.5278 [hep-ex].

[21] M. Anselmino, M. Boglione, U. D’Alesio, A. Kotzinian, F. Murgia, A. Prokudin and S. Melis, Nucl. Phys. Proc. Suppl. 191, 98 (2009).

[22] J. Soffer, Phys. Rev. Lett. 74, 1292 (1995).

[23] J. C. Collins, S. F. Heppelmann and G. A. Ladinsky, Nucl. Phys. B 420, 565 (1994).

[24] A. Airapetian et al. [HERMES Collaboration], JHEP 0806, 017 (2008).

[25] C. Adolph et al. [COMPASS Collaboration], Phys. Lett. B 713, 10 (2012).

[26] A. Vossen et al. [Belle Collaboration], Phys. Rev. Lett. 107, 072004 (2011).

[27] A. Bacchetta, A. Courtoy and M. Radici, JHEP 1303, 119 (2013). 